\title{
Functions of Chitosan-Ferulic Acid Salt for Prevention of Hypertension
}

\author{
Yoshifumi Murata $^{1 *}$, Kumiko Nagaki ${ }^{1}$, Kyoko KofusI $^{1}$ and Toshihiro KIsHi ${ }^{2}$ \\ ${ }^{1}$ Faculty of Pharmaceutical Science, Hokuriku University, Ho-3, Kanagawa-machi, Kanazawa 920-1181, Japan \\ ${ }^{2}$ Nippon Suisan, Ltd., Otemachi, Chiyoda-ku, Tokyo 100-8686, Japan
}

Received April 14, 2010; Accepted May 31, 2010

\begin{abstract}
A chitosan (CS)-ferulic acid (FA) salt was prepared. FA release from CS-FA and bile acid (BA) adsorption by CS-FA were investigated in vitro. CS-FA released FA into artificial gastric juice or a medium containing BA. Two functions were recognized on alginate or pectin gel beads containing CS-FA: FA release and BA adsorption. Anti-hypertensive effects of CS, FA or CS-FA on hypertensive rats were tested. In rats given a diet containing CS-FA or FA, elevation of systolic blood pressure was suppressed compared to the control diet group, but no difference in food intake or body weight was observed between groups.
\end{abstract}

Keywords: ferulic acid, chitosan, anti-hypertensive effect, bile acid adsorption, alginate gel bead

\section{Introduction}

Polyphenolic substances exist widely in fruits, vegetables and grains. Some of them have been known as antioxidant compounds that show potentially protective effects against hepatic and heart diseases, as well as Alzheimer's disease (Hamaguchi et al., 2009; Rukkumani et al., 2004). A polyphenol, trans-4-hydroxy-3-metoxy-cinnamic acid (ferulic acid, FA), has been used as an anti-oxidizing agent for foodstuffs (Alamed et al., 2009). Its physiological action to mitigate lifestyle-related diseases, such as hypertension, hyperlipidemia, and diabetes, has been noted (Ohnishi et al., 2004; Suzuki et al., 2002; Wang et al., 2004). Oral administration of FA was found to reduce the level of plasma cholesterol and triglyceride in rats (Balasubashini et al., 2003). These results show that intestinal absorption of FA is associated with an $\mathrm{H}^{+}$-driven transport system (Zhao et al., 2004). FA has commonly been taken by individuals with the habit of drinking coffee (Renouf et al., 2010).

A natural cationic polysaccharide, chitosan (CS), has also been used in the food industry. It is a dietary fiber that decreases plasma cholesterol by daily uptake (Kaats et al., 2006; Yao et al., 2008). The main mechanisms of the hypocholesterolemic effect is through the adsorption of dietary fat by CS and through adsorption of bile acid (BA) excreted in the intestinal tract (Baker et al., 2009; Shields et al., 2003). In a mouse study, the low bile acid-binding capabilities of CS

*To whom correspondence should be addressed.

E-mail: y-murata@hokuriku-u.ac.jp have been shown (Bennekum et al., 2005). We reported that CS salts adsorb BA in vitro, but this phenomenon has not been observed in the case of CS itself (Murata et al., 2006). Therefore, following oral administration of CS, the cationic polysaccharide might turn into $\mathrm{HCl}$ salt in the human stomach and subsequently bind BA through an ion-exchange reaction.

Although FA is relatively hydrophilic (Sohn and $\mathrm{Oh}$, 2003), it is a weaker acid with more limited water solubility than either acetic or lactic acid. Nevertheless, FA might induce formation of a CS salt because CS was shown to change the salt in a suspension of 2-(4-chlorophenoxy)-2methylpropionic acid (Murata et al., 2004). Moreover, the electrostatic complex formed between FA and CS (i.e., CSFA) might be able to induce two functions, FA release and $\mathrm{BA}$ adsorption without changing the $\mathrm{CS}-\mathrm{HCl}$ salt by gastric juice.

In the present study, we attempted to prepare CS-FA and investigated FA release from CS-FA and BA adsorption by the salt in vitro. FA released in guts might be absorbed and exhibit the pharmacological action if CS-FA is administered orally for subsequent transfer to the gastrointestinal tract. Therefore, CS-FA was introduced into the feed of spontaneously hypertensive rats (SHR) to investigate its anti-hypertensive effect. In addition, as CS-FA is a fine powder making it difficult to swallow, we devised a form of the salt for swallowing, by incorporating it into calcium-induced alginate gel beads (Alg-Ca) or pectin gel beads. The capabilities of these forms to release FA and absorb BA were examined. 


\section{Materials and Methods}

For use in this study, FA was purchased from Tsuno Food Ind. Co. Ltd. (Wakayama, Japan). CS (fine powder, degree of deacetylation: 75-85\%) was obtained from Kimitsu Chemical Industries Co. Ltd. (Tokyo, Japan) and pectin (GENU pectin LM-102AS) was purchased from Sanshou Co. (Tokyo, Japan). In addition, $\mathrm{N}^{\mathrm{G}}$-nitro-L-arginine methyl ester hydrochloride (L-NAME) was purchased from Nacalai Tesque Inc. (Kyoto, Japan). All other chemicals containing BA were of reagent grade. As an artificial gastric juice, JP XIV 1st medium ( $\mathrm{pH}$ 1.2) was used.

Preparation of CS-FA CS (1 g), FA (0.5 g) and deionized water $(23.5 \mathrm{~g})$ were mixed for 1 day at room temperature. The suspension was then centrifuged at $3000 \mathrm{rpm}$ for 10 min. The precipitate was collected and washed three times with ethanol, dried on a dish, and then desiccated under a vacuum in the presence of $\mathrm{P}_{2} \mathrm{O}_{5}$. The obtained powder was called CS-FA (2:1). CS-FA (1:1) prepared with CS (1 g) and FA (1 g), and CS-FA (1:2) prepared with CS (1 g) and FA (2 g) were obtained using the same method.

$X$-ray diffractometry Powder X-ray diffractometry was carried out using an automatic diffractometer (MXP3; MAC Science Ltd., Yokohama, Japan) with a voltage of $40 \mathrm{kV}$ and a current of $20 \mathrm{~mA}$. The scanning rate was $2 \% / \mathrm{min}$ over a 2 theta range of $5-100^{\circ}$. The results of X-ray diffraction were interpreted using a computer program (Bruker AXS K.K., Yokohama, Japan).

Test of FA release from $C S-F A$ and $B A$ adsorption to $C S$ $F A \quad$ Either $15 \mathrm{~mL}$ of artificial gastric juice, deionized water or a $2 \mathrm{mmol} / \mathrm{L}$ BA solution $(\mathrm{pH}$ 5.9-6.0) was placed into a vial tube and maintained at $37^{\circ} \mathrm{C}$. Then, $15 \mathrm{mg}$ of CS-FA was added to the tubes and shaken. After $2 \mathrm{~h}$, the FA released from CS-FA or BA adsorbed to CS-FA was measured using HPLC. All tests were performed in triplicate. The HPLC system had an LC-6A pump (Shimadzu Co., Kyoto, Japan), a packed column $(150 \mathrm{~mm} \times 4.6 \mathrm{~mm}$, Mightysil RP-18 GP; Kanto Chemical Co., Tokyo, Japan), and a SPD-6A UV detector (Shimadzu Co.). The HPLC for determination of FA was conducted at ambient temperature using an eluent containing $10 \mathrm{mmol} / \mathrm{L}$ phosphate buffer ( $\mathrm{pH} \mathrm{4.7)}$ and methanol $(8: 2)$ at a flow rate of $0.8 \mathrm{~mL} / \mathrm{min}$. The detector wavelength was set as $260 \mathrm{~nm}$. HPLC for the determination of BA was conducted at ambient temperature using an eluent containing methanol, $30 \mathrm{mmol} / \mathrm{L}$ phosphate buffer $(\mathrm{pH} \mathrm{3.4)}$, and acetonitrile $(6: 3: 1)$ at a flow rate of $0.8 \mathrm{~mL} / \mathrm{min}$. The detector wavelength was set as $230 \mathrm{~nm}$. The amount of BA adsorbed to CS-FA was calculated based on the amount of BA added and that remaining at sampling time.

\section{Preparation of gel beads containing CS-FA}

We prepared Alg-Ca (hydrogel beads) containing CS-
Table 1. Composition of test diets.

\begin{tabular}{lcccc}
\hline \multicolumn{5}{c}{ Components (\%) } \\
Diet & CRF-1 & CS-FA(2:1) & CS & FA \\
\hline Control & 100 & 0 & 0 & 0 \\
CS-FA & 90 & 10 & 0 & 0 \\
CS & 92.1 & 0 & 7.9 & 0 \\
FA & 97.9 & 0 & 0 & 2.1 \\
\hline
\end{tabular}

FA as follows: $1 \mathrm{~g}$ of $\mathrm{CS}$ was dispersed in $9 \mathrm{~g}$ of $1(\mathrm{w} / \mathrm{w}) \%$ sodium alginate solution with agitation. This solution (2 g) was dropped into $10 \mathrm{~mL}$ of $0.1 \mathrm{~mol} / \mathrm{L} \mathrm{CaCl}_{2}$, then left to stand at room temperature for $1 \mathrm{~h}$. The spherical beads were transferred to $10 \mathrm{~mL}$ of distilled deionized water containing $0.1 \mathrm{~g}$ of FA, which was incubated at $37^{\circ} \mathrm{C}$ for a day. Pectin gel beads containing CS-FA was also prepared with $2(\mathrm{w} / \mathrm{w}) \%$ pectin instead of sodium alginate using the method described above. Dried gel beads were obtained by drying the hydrogel at for $8 \mathrm{~h}$ on a dish, followed by vacuum treatment in a desiccator in the presence of $\mathrm{P}_{2} \mathrm{O}_{5}$.

Test of FA release and $B A$ adsorption by gel beads containing CS-FA Gel beads containing CS-FA corresponding to $2 \mathrm{~g}$ of hydrogel were added to $15 \mathrm{~mL}$ of $2 \mathrm{mmol} / \mathrm{L} \mathrm{BA}$ solution incubated at $37^{\circ} \mathrm{C}$. A $0.2-\mathrm{mL}$ aliquot of the solution was removed periodically to determine FA released and BA taken up into the gel beads using HPLC as described.

Animal study The powdered feed given to the rats was a certified diet (CRF-1; Oriental Yeast Co. Ltd., Japan). The composition of the diet containing CS, FA or CS-FA (2:1) is shown in Table 1. Each ration was mixed well and given to rats after sifting through a sieve $(710 \mu \mathrm{m})$.

The Animal Care and Use Committee of Hokuriku University approved the experimental protocol. Male SHR/Izm rats (4-weeks-old, 60-100 g; Sankyo Lab.) were housed individually in stainless-steel wire-bottomed cages in a temperature-controlled room. They were allowed free access to food and water for 1 week. For the following 5 weeks, $0.003 \% \mathrm{~L}$ NAME was used as drinking water (Pechanoval et al., 1999). The food intake of each rat was measured daily and body weight was measured weekly. Systolic blood pressure (SBP) was measured weekly using the tail-cuff method in conscious SHR placed in a holder using an automatic blood pressure monitoring system (MK-2000; Muromachi Kikai Co. Ltd., Japan). Where necessary, data were compared using ANOVA (Tukey's test) and their difference was inferred as significant when $p<0.01$.

\section{Results and Discussion}

Figure 1 shows the X-ray diffraction patterns obtained from CS-FA, CS, FA and their physical mixtures; FA exhibited a characteristic crystalline compound pattern of dif- 

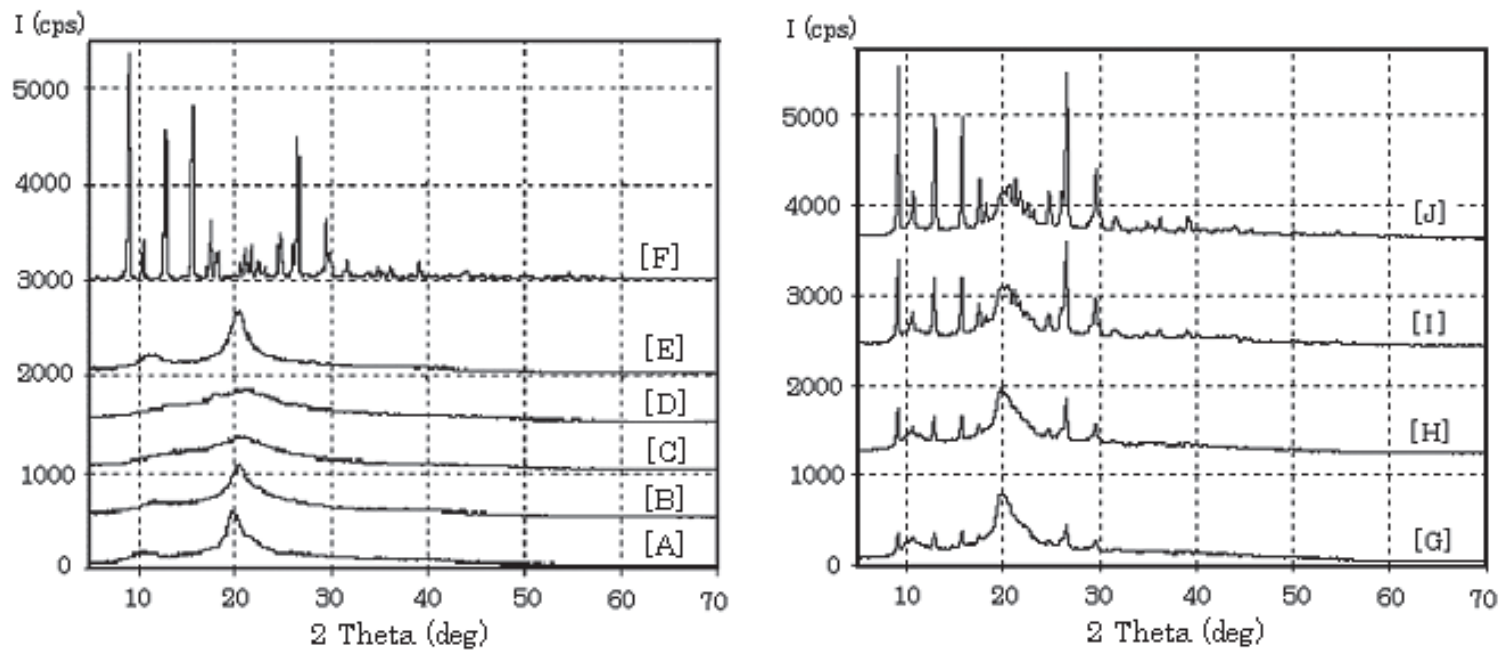

Fig. 1. X-ray diffractograms.

[A] CS, [B] CS-FA(2:1), [C] CS-FA(1:1), [D] CS-FA(1:2), [E] CS treated with deionized water, [F] FA, [G] mixture of FA and CS (1:10), [H] mixture of FA and CS (1:5), [I] mixture of FA and CS (1:2), [J] mixture of FA and CS (1:1).

fraction. The pattern was also apparent in the mixture of CS and FA. On the other hand, CS-FA showed a pattern which lacked the characteristic diffraction peaks of FA. This result shows that the crystal form of FA is only slightly present in CS-FA. The broad peak of CS at $20^{\circ}$ decreased according to the increment of FA amount in the CS-FA preparation. The peak disappearance was not recognized in the case of CS treated with deionized water and not contain FA or in the case of the physical mixtures.

CS-FA released FA into artificial gastric juice: $0.434 \pm$ $0.006 \mathrm{mmol} / \mathrm{g} \mathrm{CS}-\mathrm{FA}(2: 1)$; the amount was $0.058 \pm 0.002$ $\mathrm{mmol} / \mathrm{g}$ CS-FA(2:1) in deionized water. This result indicates that FA mainly exists as an ion pair with the amino group of CS in CS-FA. The FA is liberated from CS-FA when hydrogen chloride is exchanged for FA on the polymer. The CS-FA also released FA into the medium containing BA as it simultaneously adsorbed BA as shown in Figure 2. In the case of taurocholate (TCA), the amount of FA released was $0.261 \pm$ $0.001 \mathrm{mmol} / \mathrm{g} \mathrm{CS}-\mathrm{FA}(2: 1)$ and the amount of TCA adsorbed was $0.280 \pm 0.002 \mathrm{mmol} / \mathrm{g} \mathrm{CS}-\mathrm{FA}(2: 1)$.

A sample of Alg-Ca prepared in this study theoretically contains about $0.1 \mathrm{~g}$ of CS, which was converted to CS-FA within the gel matrix by incubation in FA suspension. Two functions of CS-FA were recognized on Alg-Ca containing CS-FA: the release of FA and the adsorption of BA. Figure 3 shows when hydrogel beads $(2 \mathrm{~g})$ were shaken in $15 \mathrm{~mL}$ of $2 \mathrm{mmol} / \mathrm{L}$ TCA solution, it immediately took up TCA (2 h, $24.7 \pm 0.2 \mu \mathrm{mol})$. The uptake was observed for other BAs such as glycocholate $(25.9 \pm 0.2 \mu \mathrm{mol})$, taurodeoxycholate $(28.2 \pm 0.3 \mu \mathrm{mol})$, and taurochenodeoxycholate $(28.1 \pm 0.1$

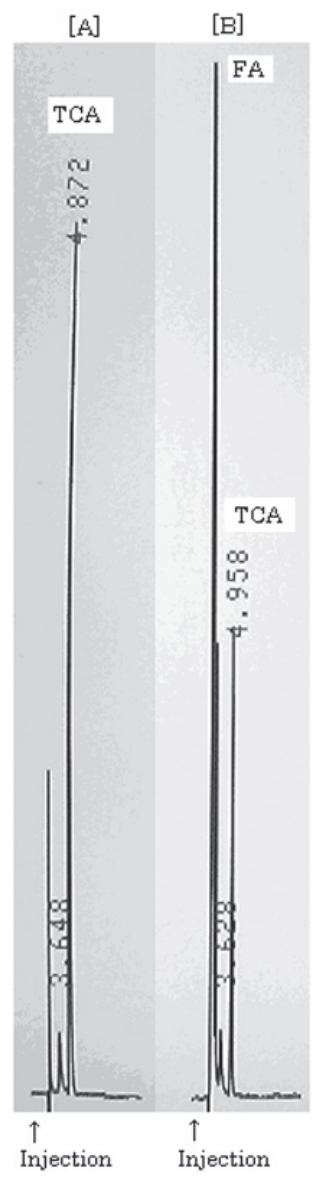

Fig. 2. Chromatograms of TCA obtained from HPLC determination.

[A] TCA solution, [B] TCA solution with CS-FA. 

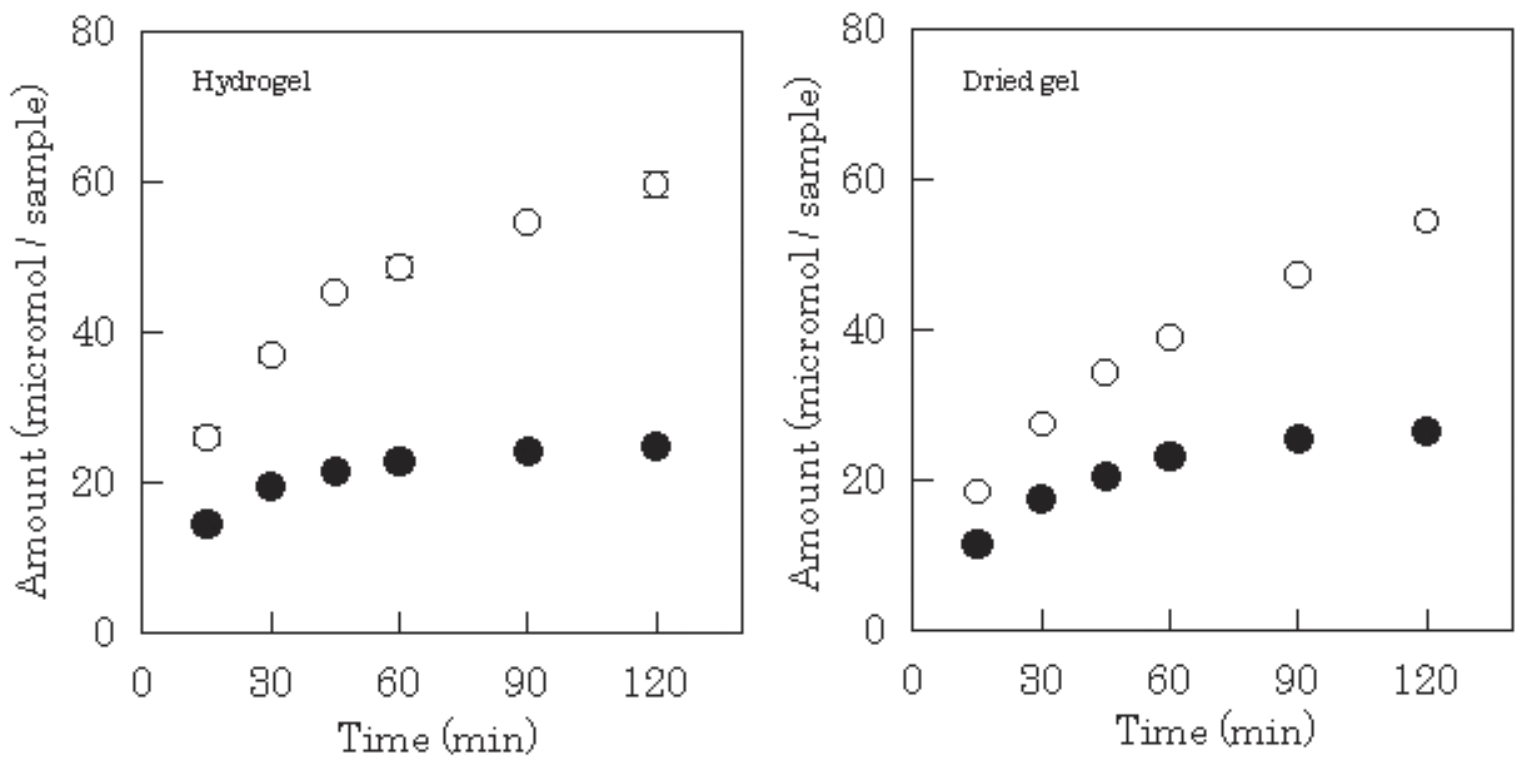

Fig. 3. FA release (०) from Alg-Ca containing CS-FA and uptake of TCA (•). Values are means \pm SD of triplicate analyses.

$\mu \mathrm{mol})$ after $2 \mathrm{~h}$. In all cases, the upper $80 \%$ of BA dissolved in the solution was taken up; the uptake amount increased according to the increment of BA concentration (data not shown). On the other hand, the gel beads gradually released FA: the amount released was $59.7 \pm 1.6 \mu \mathrm{mol}$ after $2 \mathrm{~h}$. Similar patterns of FA release and TCA uptake were apparent in the case of dried Alg-Ca. The FA existed not only as CS-FA, but as a free inner gel matrix. Both types were released when Alg-Ca was immersed in a TCA solution.

Therefore, the amount of FA released was greater than that of TCA taken up into Alg-Ca. An identical phenomenon was recognized when pectin beads containing CS-FA were used as the vehicle. The amount of TCA adsorbed after $2 \mathrm{~h}$ was $25.5 \pm 0.3 \mu \mathrm{mol} / \mathrm{sample}$ and the amount of FA released was $60.2 \pm 4.1 \mu \mathrm{mol} / \mathrm{sample}$ in the case of calcium-induced pectin beads.

When administered orally, FA might be released from CS-FA and absorbed in the gastrointestinal tract. Reportedly, FA transport occurs via an $\mathrm{H}^{+}$-dependent system in Caco-2 cells (Itagaki et al., 2005). Moreover, supplementation with FA has shown physiological actions like a protective effect on hyperlipidemia in diabetic rats (Zhao et al., 2004). In the present study, CS-FA, FA or CS was mixed into the powder diet given to SHR for 5 weeks. No changes in food intake (about $19 \mathrm{~g} / \mathrm{d}$ ) were observed in any diet group, and no apparent difference in body weight changes resulted from the various administered foods (Figure 4 ). In the case of the control diet group, SBP was initially $125 \pm 12 \mathrm{mmHg}(n=5)$ and increased gradually to $263 \pm 13 \mathrm{mmHg}$ after 5 weeks (Figure 5). In the case of the CS-FA diet group (theoretically about $1.9 \mathrm{~g}$ of CS-FA was taken up per day), SBP was initially $126 \pm 8 \mathrm{mmHg}$ and its elevation was suppressed compared to the control group; it was $188 \pm 12 \mathrm{mmHg}$ after 5 weeks. FA is known to be beneficial for preventing the elevation of SBP (Suzuki et al., 2002), which was confirmed based on the identical phenomenon being observed with the FA diet group, while no pronounced effect was recognized in rats in the CS diet group. These results show that the effect might be mainly attributed to FA released from CS-FA into the gastrointestinal tract.

Both CS and FA have been used as food additives. CS-FA was prepared using a simple method, with two functions: FA release and BA adsorption. These functions are also expected in the polysaccharide gel matrix, which is suitable for oral administration. As CS-FA could release FA into artificial gastric juice or bile acid solution, FA might be released into the stomach and intestine when CS-FA is administered orally. The FA is known to be absorbed into the gastrointestinal tract and to show a pharmacological action. Actually, elevation of blood pressure in SHR was suppressed by the daily intake not only of FA, but also of CS-FA. On the other hand, CS-FA might change the $\mathrm{CS}-\mathrm{HCl}$ salt in the stomach, allowing the salt to adsorb BA as well as CS-FA (Ylitalo, et al., 2002).

In the present study, L-NAME was given to SHR as drinking water to generate malignant hypertension (Takahashi, et al., 2007). Development of a preparation that does 

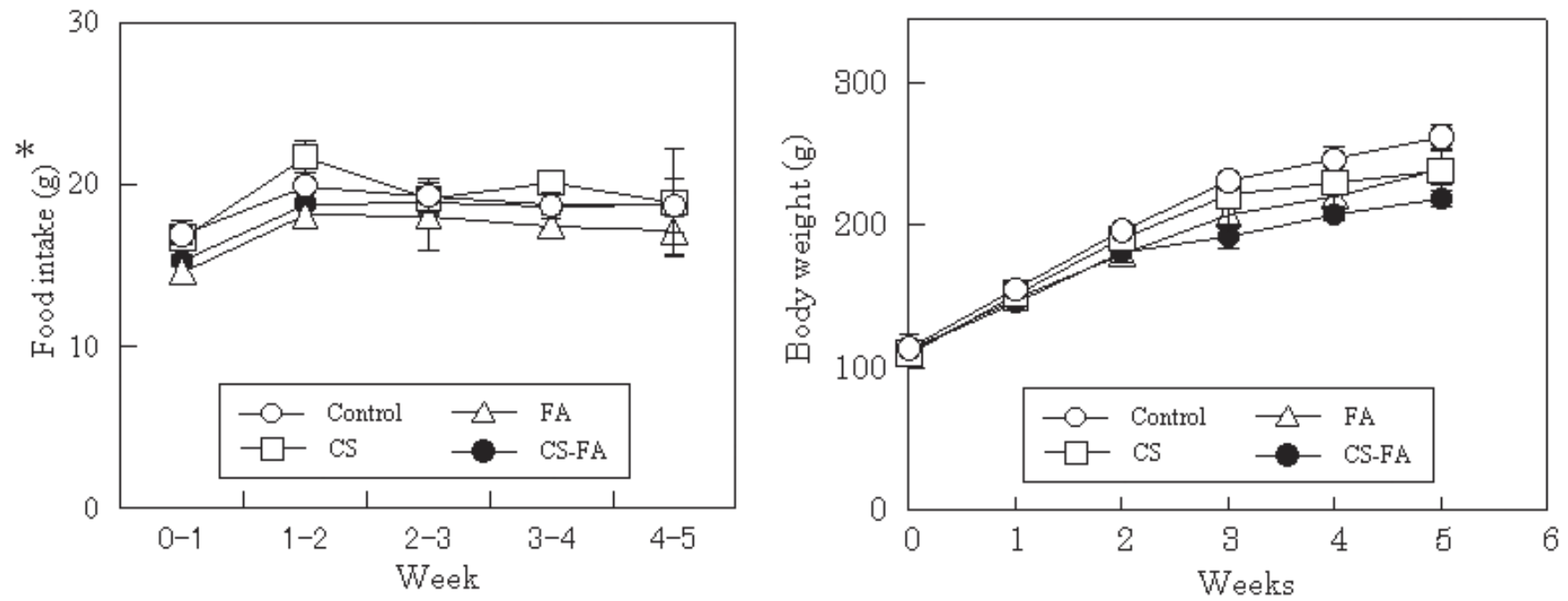

Fig. 4. Change in food intake and body weight of SHR/Izm rat $(n=5)$.

*Food intake is the average of one week.

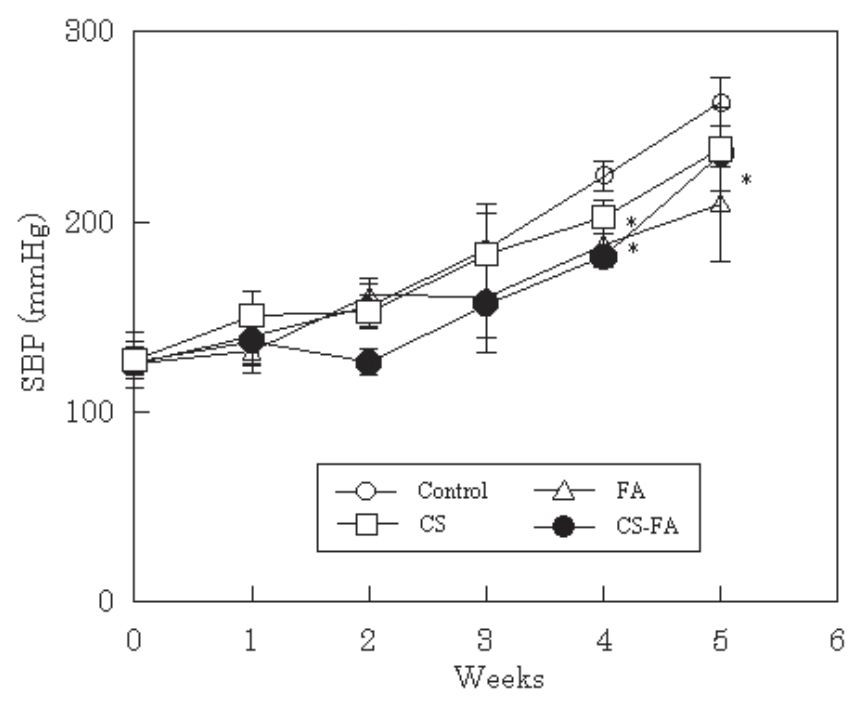

Fig. 5. Effect of diet on change in $\operatorname{SBP}(n=5)$. $* P<0.01$, compared with the control.

not impart harmful effects is essential for prevention of lifestyle-related diseases such as hypertension. Therefore, we shall investigate further the preventive effect of CS-FA on such diseases.

\section{References}

Alamed, J., Chaiyasit, W., McClements, D.J. and Decker, E.A. (2009). Relationships between free radical scavenging and antioxidant activity in foods. J. Agric. Food Chem., 57, 2969-2976.

Baker, W.L., Tercius, A., Anglade, M., White, C.M. and Coleman, C.I. (2009). A meta-analysis evaluating the impact of chitosan on serum lipids in hypercholesterolemic patients. Ann. Nutr. Metab., $\mathbf{5 5}, 368-374$.
Balasubashini, M.S., Rukkumani, R. and Menon, V.P. (2003). Protective effects of ferulic acid on hyperlipidemic diabetic rats. Acta, Diabetol., 40, 118-122.

Hamaguchi, T., Ono, K., Murase A. and Yamada M. (2009). Phenolic compounds prevent Alzheimer's pathology through different effects on the amyloid-beta aggregation pathway. Am. J. Pathol., 175, 2557-2565.

Itagaki, S., Kobayashi, Y., Otsuka, Y., Kubo, S. Kobayashi, M., Hirano, T. and Iseki, K. (2005). Food-drug interaction between ferulic acid and nateglinide involving the fluorescein $/ \mathrm{H}^{+}$cotransport system. J. Agric. Food Chem., 53, 2499-2502.

Kaats, G.R., Michalek, J.E. and Preuss, H.G. (2006). Evaluating efficacy of a chitosan product using a double-blinded, placebocontrolled protocol. J. Am. Coll. Nutr., 25, 389-394.

Murata, Y., Hirai, D., Kofuji, K., Miyamoto, E. and Kawashima, S. (2004). Properties of an alginate gel bead containing a chitosandrug salt. Biol. Pharm. Bull., 27, 440-442.

Murata, Y., Nagaki, K., Kofuji, K., Sanae, F., Kontani, H. and Kawashima, S. (2006). Adsorption of bile acid by chitosan salts prepared with cinnamic acid and analogue compounds. J. Biomater. Sci. Polym. Ed., 17, 781-789.

Ohnishi, M., Matsuo, T., Tsuno, T., Hosoda, A., Nomura, E., Taniguchi, H., Sasaki, H. and Morishita, H. (2004). Antioxidant activity and hypoglycemic effect of ferulic acid in STZ-induced diabetic mice and KK-Ay mice. Biofactors, 21, 315-319.

Pechanova, O., Bernatova, I., Pelouch, V. and Babal, P. (1999). LNAME-induced protein remodeling and fibrosis in the rat heart. Physiol. Res., 48, 353-362.

Renouf, M., Guy, P.A., Marmet, C., Fraering, A.L., Longet, K., Moulin, J., Enslen, M., Barron, D., Dionisi, F., Cavin, C., Williamson, G. and Steiling, H. (2010). Measurement of caffeic acid 
and ferulic acid equivalents in plasma after coffee consumption: Small intestine and colon are key sites for coffee metabolism. Mol. Nutr. Food Res., 54, 1-7.

Rukkumani, R., Aruna, K., Suresh, V.P. and Padmanabhan, M.V. (2004). Hepatoprotective role of ferulic acid: a dose-dependent study. J. Med. Food, 7, 456-461.

Shields, K.M., Smock, N., McQueen, C.E. and Bryant, P.J. (2003). Chitosan for weight loss and cholesterol management. Am. J. Health Syst. Pharm., 60, 1310-1312.

Sohn, Y.T. and Oh, J.H., (2003). Characterization of physicochemical properties of ferulic acid. Arch. Pharm. Res., 26, 1002-1008.

Suzuki, A., Kagawa, D., Fujii, A., Ochiai, R., Tokimitsu, I. and Saito, I. (2002). Short- and long-term effects of ferulic acid on blood pressure in spontaneously hypertensive rats. Am. J. Hypertens., 15, 351-357.

Takahashi, T., Ono, H., Ono, Y., Ishimitsu, T. and Matsuoka H. (2007). Combination therapy with telmisartan and spironolactone alleviates L-NAME exacerbated nephrosclerosis with an increase in PPAR- $\gamma$ and decrease in TGF- $\beta_{1}$. Int. Heart J., 48, 637-647.

Van Bennekum, A.M., Nguyen, D.V., Schulthess, G., Hauser, H. and Philips, M.C. (2005). Mechanism of cholesterol-lowering effects of dietary insoluble fibres: relationships with intestinal and hepatic cholesterol parameters. Br. J. Nutr., 94, 331-337.

Wang, B., Ouyang, J., Liu, Y., Yang, J., Wei, L., Li, K. and Yang, H. (2004). Sodium ferulate inhibits atherosclerogenesis in hyperlipidemia rabbits. J. Cardiovasc. Pharmacol., 43, 549-554.

Yao, H.T., Huang, S.Y. and Chiang, M.T. (2008). A comparative study on hypoglycemic and hypocholesterolemic effects of high and low molecular weight chitosan in streptozotocin-induced diabetic rats. Food Chem. Toxicol., 46, 1525-1534.

Ylitalo, R., Lehtinen, S., Wuolijoki, E., Ylitalo, P. and Lehtimaki, T. (2002). Cholesterol-lowering properties and safety of chitosan. Arzneimittelforschung, 52, 1-7.

Zhao, Z., Egashira, Y. and Sanada, H. (2004). Ferulic acid is quickly absorbed from rat stomach as the free form and the conjugated mainly in liver. J. Nutr., 134, 3083-3088. 